\title{
Effect of Antenna Mutual Coupling on MIMO Channel Estimation and Capacity
}

\author{
Xia Liu and Marek E. Bialkowski \\ School of ITEE, The University of Queensland, Brisbane, QLD 4072, Australia \\ Correspondence should be addressed to Xia Liu, xialiu@itee.uq.edu.au
}

Received 2 December 2009; Accepted 17 January 2010

Academic Editor: Hon Tat Hui

Copyright ( $) 2010$ X. Liu and M. E. Bialkowski. This is an open access article distributed under the Creative Commons Attribution License, which permits unrestricted use, distribution, and reproduction in any medium, provided the original work is properly cited.

\begin{abstract}
This paper describes investigations into the antenna mutual coupling $(\mathrm{MC})$ effect on channel estimation and capacity of a multipleinput multiple-output (MIMO) wireless communication system. The presented investigations close the gap existing in the previous works which assessed the effect of mutual coupling on MIMO capacity under the assumption of availability of perfect channel state information (CSI) at the receiver. The new approach assumes that the perfect CSI is not available due to channel estimation errors. The investigations are carried out for different spacing between array antenna elements producing a varying effect of mutual coupling on the channel estimation and the resulting MIMO channel capacity.
\end{abstract}

\section{Introduction}

It has been shown in recent works that a wireless communication system can achieve an increased capacity by using multiple element antennas at transmitter and receiver by applying a suitable signal processing scheme [1]. This unconventional wireless communication system is called a multiple-input multiple-output (MIMO) system. It offers an increased capacity without the need of increasing an operational bandwidth.

In order to realize the advantages of MIMO, two conditions have to be satisfied. One requires the presence of a rich scattering environment, and the other one entails accurate channel state information (CSI) to be available at the receiver [2]. The rich scattering environment is necessary to support the formation of statistically independent virtual channels over which the parallel data transmission can take place. The lack of (spatial) correlation between the virtual channels leads to the increased MIMO capacity. The availability of accurate CSI is required to decode the received signal and to practically reach the MIMO capacity [1-5]. In turn, an inaccurate CSI leads to an increased bit error rate (BER) that translates into a degraded capacity of the system [6-8].
It is known that the finite antenna spacing in array antennas introduces spatial correlation. This finite spacing is also responsible for mutual coupling which adversely affects signal transmission and reception due to the resulting antenna impedance mismatch. The mutual coupling effect is especially pronounced in tightly spaced arrays. Because of a considerable demand for compact size mobile station (MS) terminals, the effect of mutual coupling cannot be neglected and thus has to be taken into account while assessing the MIMO link performance. The problem of mutual coupling in MIMO systems for the case of peerto-peer communication has been addressed via simulations and measurements in [9-15]. It has been shown that the mutual coupling may improve the channel capacity when the spacing of antenna elements in the transmit and receive array antennas is between 0.2 to $0.4 \lambda$ (carrier wavelength). The reason is that in this case the mutual coupling decreases the spatial correlation and increases the channel effective degree of freedom (EDOF). However, the results obtained for MIMO capacity were based on the assumption that the perfect channel state information (CSI) was available at least at the receiver end. In practical cases, perfect CSI is not achievable due to channel estimation errors. To function 
properly, the MIMO system has to estimate properties of the channel before decoding the received data.

Obtaining an accurate CSI can be accomplished using suitable channel estimation methods. The methods based on the use of training sequences, known as the trainingbased channel estimation methods, are the most popular. In $[16,17]$, several training-based methods including least square (LS) method, scaled least square (SLS) method and minimum mean square error (MMSE) method have been investigated. It has been shown that the accuracy of the investigated training-based channel estimation methods is influenced by the transmitted signal to noise ratio (SNR) in the training mode, and the number of antenna elements at the transmitter and receiver. In particular, it has been pointed out that when the transmitted SNR and the number of antenna elements are fixed, the SLS and MMSE methods offer better performance than the LS method. In [18-20], it has been shown that if the training-based SLS or MMSE channel estimation methods are used to estimate CSI, their performance can be improved when the channels exhibit a higher spatial correlation level. According to [9-15], when the antenna element spacing is within $0.2 \lambda$ and $0.4 \lambda$, the mutual coupling can decrease the spatial correlation level. Therefore in such cases, the accuracy of training-based channel estimation methods is compromised. From these considerations one can see a tradeoff between the accuracy of channel estimation and the resulting capacity when the MIMO system operates under mutual coupling conditions. When the correlation increases, the channel estimation error becomes smaller. However, at the same time the increased correlation is responsible for decreasing the effective degree of freedom thus the MIMO channel capacity.

In this paper, the overall effect of antenna mutual coupling on performance of an MIMO wireless system is investigated. The channel capacity that provides the overall measure of MIMO system performance is determined assuming an imperfect CSI is available at the receiver. A closed-form mathematical expression for the MIMO channel capacity that includes channel estimation errors is derived. The effects of mutual coupling on channel estimation and resulting capacity are investigated via computer simulations using a suitable MIMO channel model.

The rest of the paper is organized as follows. Section 2 introduces MIMO channel and its capacity. Also described in this section are the training-based MIMO channel estimation methods, expressions for MIMO capacity including channel estimation errors, and the Kronecker model [21, 22] of MIMO channel. Section 3 describes the mathematical model for the antenna mutual coupling. Section 4 describes computer simulation results for the MIMO system capacity under the assumption of perfect CSI, as well as when an imperfect CSI due to estimation errors is available at the receiver. Section 5 concludes the paper.

\section{MIMO System}

2.1. MIMO Channel Capacity. Generally, a wireless communication channel is described by the relationship between the input signal $S$ and output signal $Y$. Assuming that $\hat{S}$ is a set of signals, $p(s)$ is the probability of $S$ given $s$, the entropy or a measure of uncertainty of $S$ can be written as,

$$
H(S)=E\{I(s)\}=-\sum_{s \in \hat{S}} p(s) \log p(s) .
$$

in which $I(s)$ is the self-information which is the entropy contribution of an individual message; $E\{\cdot\}$ stands for the expected value.

Similarly, the entropy of $Y$ given $y$ can be expressed as,

$$
H(Y)=E\{I(y)\}=-\sum_{y \in \hat{Y}} p(y) \log p(y) .
$$

The joint entropy of $S$ and $Y$ is given as,

$$
H(S, Y)=E\{-\log p(s, y)\}=-\sum_{s, y} p(s, y) \log p(s, y) .
$$

The conditional entropy (or conditional uncertainty) of $S$ given $Y$ is expressed as,

$$
\begin{aligned}
H(S \mid Y) & =E\{H(S \mid y)\}=-\sum_{y \in Y} p(y) \sum_{s \in S} p(s \mid y) \log p(s \mid y) \\
& =-\sum_{s, y} p(s, y) \log \frac{p(s, y)}{p(y)} .
\end{aligned}
$$

The conditional entropy gives the uncertainty about the channel input after the channel output has been observed. In this case, the uncertainty of the channel input can be expressed as $H(X)-H(X \mid Y)$. This is commonly known as the mutual information of the channel which is given by,

$$
I(S ; Y)=H(X)-H(X \mid Y)=\sum_{y} \sum_{s} p(s, y) \log _{2}\left[\frac{p(y \mid s)}{p(y)}\right] .
$$

The mutual information that can be optimized in terms of $p(s)$ is independent of the channel. Because the wireless communication channel capacity is defined as the maximum mutual information, then the channel capacity can be determined from,

$$
C=\max _{p(s)} I(S ; Y)
$$

In a narrow band MIMO wireless communication system, the channel is described by a complex matrix $H$. The relationship between the input signal $S$ and the output signal $Y$ can be expressed as,

$$
Y=H S+N
$$

where $N$ is the Gaussian noise with the property that $R_{N}=$ $E\left\{N N^{H}\right\}=\sigma_{n}^{2} \cdot \sigma_{n}^{2}$ is the noise power. The $S$ is transmitted random i.i.d input signal having the property that $Q=$ $E\left\{S S^{H}\right\}$ and $\operatorname{trace}(Q)$ is equal to the total transmit power $P_{s}$. The transmitted signal to noise ratio (SNR) is $\rho=P_{s} / \sigma_{n}^{2}$. 
The upper bond mutual information of MIMO can be written as,

$$
\begin{aligned}
I(S ; Y) & \leq I(S ; Y \mid H) \\
& =E\left\{\log _{2} \operatorname{det}\left[\pi e\left(H Q H^{H}+R_{N}\right)\right]\right\}-\log _{2} \operatorname{det}\left(\pi e R_{N}\right) \\
& =E\left[\log _{2}\left(\frac{\operatorname{det}\left(H Q H^{H}+R_{N}\right)}{\operatorname{det}\left(R_{N}\right)}\right)\right] \\
& =E\left\{\log _{2} \operatorname{det}\left(I_{M_{r}}+\frac{Q}{\sigma_{n}^{2}} H H^{H}\right)\right\} .
\end{aligned}
$$

Assuming the number of transmitting and receiving antennas is the same $\left(M_{t}\right.$ equal to $\left.M_{r}\right)$ and the transmitted power is equally allocated to the transmitting antennas, (8) turns into

$$
\begin{aligned}
I(S ; Y \mid H) & =E\left\{\log _{2} \operatorname{det}\left(I_{M_{r}}+\frac{P_{s}}{N_{t} \sigma_{n}^{2}} H H^{H}\right)\right\} \\
& =E\left\{\log _{2} \operatorname{det}\left(I_{M_{r}}+\frac{\rho}{N_{t}} H H^{H}\right)\right\} .
\end{aligned}
$$

The upper bound ergodic capacity can be written as,

$$
C=\max _{Q \leq P_{s}} C: E\left\{\log _{2} \operatorname{det}\left(I_{M_{r}}+\frac{\rho}{N_{t}} H H^{H}\right)\right\} .
$$

An alternative expression for the capacity can be obtained by decomposing the MIMO channel into $n=\min \left(M_{r}, M_{t}\right)$ equivalent single input single output (SISO) subchannels. The gains of each subchannel can be represented by eigenvalues of channel correlation matrix $R_{H}=H H^{H}$ as given by(11)

$$
C=\sum_{i}^{n} \log _{2}\left(1+\rho_{i} \lambda_{i}\right)
$$

where $\rho_{i}$ and $\lambda_{i}$ are transmit SNR and gain on $i$ th subchannel, respectively. In this expression the overall power constraint is used expressed as $\sum_{i}^{n} \rho_{i}=\rho$ and $\lambda_{i}$ is the $i$ th eigenvalue of the channel correlation matrix.

If power is equally allocated to each subchannel, which is easy to realize in practice, then (11) can be rewritten in the form(12)

$$
C=\sum_{i}^{n} \log _{2}\left(1+\frac{\rho}{n} \lambda_{i}\right)
$$

2.2. Training-Based MIMO Channel Estimation. For training based channel estimation methods, the relationship between the received signal and the training sequence is assumed as given by:

$$
Y_{P}=H P+N,
$$

where $P$ represents the $M_{t} \times L$ complex training matrix and $L$ is the length of the training sequence.
The goal is to estimate the complex channel matrix $H$ from the knowledge of $Y$ and $P$. The transmitted power in the training mode is constrained by the following expression

$$
\|P\|_{F}^{2}=\mathrm{P}_{p},
$$

where $\mathrm{P}_{p}$ is a given power constant and $\|\cdot\|_{F}^{2}$ stands for the Frobenius norm. The training matrices are assumed to be orthogonal $[16,17]$ and the transmitted signal to noise ratio (SNR) in the training mode is set to $\rho$ equal to $\mathrm{P}_{p} / \sigma_{n}^{2}$.

Using the LS method, the estimated channel matrix can be written in the form [23]

$$
\hat{H}_{\mathrm{LS}}=Y P^{\dagger},
$$

where $\{\cdot\}^{\dagger}$ stands for pseudoinverse and $P^{\dagger}=P^{H}\left(P P^{H}\right)^{-1}$. The mean square error (MSE) of the estimated channel matrix in the LS method is given as

$$
\operatorname{MSE}_{\mathrm{LS}}=E\left\{\left\|H-\hat{H}_{\mathrm{LS}}\right\|_{F}^{2}\right\} \text { subject to }:\|P\|_{F}^{2}=\mathrm{P}_{p}
$$

According to [16, 17], the optimal training sequence satisfying (17) is given by

$$
P P^{H}=\frac{P}{M_{t}} I .
$$

The minimum value of Mean Square Error (MSE) for the LS method is obtained as

$$
\mathrm{MSE}_{\mathrm{LS}}=\frac{M_{t}^{2} M_{r}}{\rho} .
$$

From (18) one can see that the optimal performance of the LS estimator is influenced by the number of antenna elements at the transmitter and the receiver. However, the channel matrix (and thus CSI) has no effect on the minimum value of MSE.

The SLS method further reduces the estimation error that is obtained in the LS method. The improvement is given by the scaling factor $\gamma$ :

$$
\gamma=\frac{\operatorname{tr}\left\{R_{H}\right\}}{\operatorname{MSE}_{\mathrm{LS}}+\operatorname{tr}\left\{R_{H}\right\}} .
$$

The minimized value of MSE for the SLS method can be written as $[16,17]$

$$
\mathrm{MSE}_{\mathrm{SLS}}=\frac{\mathrm{MSE}_{\mathrm{LS}} \operatorname{tr}\left\{R_{H}\right\}}{\mathrm{MSE}_{\mathrm{LS}}+\operatorname{tr}\left\{R_{H}\right\}}<\mathrm{MSE}_{\mathrm{LS}} .
$$

By substituting (18) into (20), the minimum MSE is given by expression,

$$
\operatorname{MSE}_{\mathrm{SLS}}=\frac{1}{1 /\left(\operatorname{tr}\left\{R_{H}\right\}\right)+\rho /\left(M_{t}^{2} M_{r}\right)} .
$$

The estimated channel matrix obtained using the SLS method is given as $[16,17]$,

$$
\hat{H}_{\mathrm{SLS}}=\gamma \hat{H}_{\mathrm{LS}}=\frac{\operatorname{tr}\left\{R_{H}\right\}}{\mathrm{MSE}_{\mathrm{LS}}+\operatorname{tr}\left\{R_{H}\right\}} Y_{p} P^{\dagger} .
$$


Similar derivations apply for the MMSE channel estimation method. Using this method, the estimated channel matrix can be obtained from,

$$
\hat{H}_{\text {MMSE }}=Y\left(P^{H} R_{H} P+\sigma_{n}^{2} M_{r} I\right)^{-1} P^{H} R_{H} .
$$

By denoting the channel estimation error as,

$$
e=H-\hat{H}_{\mathrm{MMSE}}
$$

the MSE for the MMSE method can be expressed as

$$
\begin{aligned}
\mathrm{MSE}_{\mathrm{MMSE}} & =\operatorname{tr}\left\{R_{E}\right\} \\
& =\operatorname{tr}\left\{E\left\{e e^{H}\right\}\right\} \\
& =\operatorname{tr}\left\{\left(R_{H}^{-1}+\frac{1}{\sigma_{n}^{2} M_{r}} P P^{H}\right)^{-1}\right\} \\
& =\operatorname{tr}\left\{\left(\Lambda^{-1}+\sigma_{n}^{-2} M_{r}^{-1} Q^{H} P P^{H} Q\right)^{-1}\right\} .
\end{aligned}
$$

In (25), $Q$ is a unitary eigenvector matrix of $R_{H}$ and $\Lambda$ is a diagonal matrix with eigenvalues of $R_{H}$. They are obtained by applying the eigenvalue decomposition to $R_{H}$ :

$$
R^{H}=H^{H} H=Q \Lambda Q^{H} .
$$

2.3. MIMO Channel Capacity Including Channel Estimation Errors. In practical cases, $H$ has to be replaced by the estimated channel matrix, which carries an estimation error as described in the last section. By assuming that the channel estimation error is defined as $e$ and the estimated channel matrix is $\hat{H}$ given by

$$
\hat{H}=H+e .
$$

The received signal can accordingly be written as

$$
Y=\hat{H} S+e S+N \text {. }
$$

The variance of each element in the estimation error matrix is given as

$$
\sigma_{e, i j}^{2}=E\left\{\left(H_{i j}-\hat{H}_{i j}\right)^{2}\right\}
$$

If the channel matrix describes an uncorrelated Rayleigh channel, the elements in the estimation error matrix e are all uncorrelated identical independent distribution (i.i.d) with zero mean. In this case, the variance of each element in the error matrix shares the same value as $\sigma_{e}^{2}$; the error matrix correlation is diagonal and holds the property

$$
R_{e}=\sigma_{e}^{2} I_{\min }\left(M_{r}, M_{t}\right)
$$

Using (25), one finds

$$
\sigma_{e}^{2}=\frac{\mathrm{MSE}}{\min \left(M_{r}, M_{t}\right)}
$$

The channel capacity of MIMO system with an imperfectly known $H$ at the receiver is defined as the maximum mutual information between $Y$ and $S$ :

$$
C=\max _{\operatorname{tr}\{Q\} \leq P_{s}}\{I(S ; Y, \hat{H})\}
$$

If the transmitter does not have any knowledge of the channel, the mutual information in (32) can be written as,

$$
I(S ; Y, \hat{H})=I(S ; Y \mid \hat{H})=h(S \mid \hat{H})-h(S \mid Y, \hat{H}) .
$$

Because adding any dependent term on $Y$ does not change the entropy [24], then

$$
h(S \mid Y, \hat{H})=h(S-u Y \mid Y, \hat{H})
$$

in which $u$ is the MMSE estimator given as

$$
u=\frac{E\left\{S Y^{H} \mid \hat{H}\right\}}{E\left\{Y Y^{H} \mid \hat{H}\right\}} .
$$

Combining the above with (28), one obtains

$$
\begin{aligned}
u & =\frac{E\left\{S(\hat{H} S+e S+V)^{H} \mid \hat{H}\right\}}{E\left\{(\hat{H} S+e S+V)(\hat{H} S+e S+V)^{H} \mid \hat{H}\right\}} \\
& =\frac{Q \hat{H}^{H}}{\hat{H} Q \hat{H}^{H}+E\left\{e Q e^{H}\right\}+\sigma_{n}^{2} I_{M_{r}}} .
\end{aligned}
$$

If one assumes the special case of $M_{t}$ equal to $M_{r}$ and the transmitted signal power being equally allocated to the transmitting antennas, (36) becomes

$$
\begin{aligned}
u & =\frac{Q \hat{H}^{H}}{\hat{H} Q \hat{H}^{H}+Q \sigma_{e}^{2} I_{M_{r}}+\sigma_{n}^{2} I_{M_{r}}} \\
& =\frac{p \hat{H}^{H}}{p \hat{H} \hat{H}^{H}+p \sigma_{e}^{2} I_{M_{r}}+\sigma_{n}^{2} I_{M_{r}}}
\end{aligned}
$$

in which $p=P_{s} / M_{t}$ is the power allocated to the signal transmitted through each transmit antenna. The conditioning decreases the entropy and thus,

$$
h(S-u Y \mid \hat{H})=h(S-u Y \mid Y, \hat{H}) .
$$

Next, the following can be applied:

$$
h(S-u Y \mid \hat{H})=h(S-u Y \mid Y, \hat{H})
$$


and thus,

$$
I(S ; Y \mid \hat{H})=h(S \mid \hat{H})-h(S-u Y \mid Y, \hat{H}) .
$$

For the case of $S \mid \hat{H}$ and $(S-u Y \mid Y, \hat{H})$ having a Gaussian distribution, (40) can be expressed as [24-26],

$$
\begin{aligned}
I(S ; Y \mid \hat{H}) \\
\geq E\left\{\log _{2}[\operatorname{det}(\pi e Q)]\right\} \\
\quad-E\left\{\log _{2}\left[\operatorname{det}\left(\pi e E\left\{(S-u Y)(S-u Y)^{H} \mid \hat{H}\right\}\right)\right]\right\} \\
\geq E\left\{\log _{2}\left[\operatorname{det}\left(I_{M_{r}}+\frac{p \hat{H} \hat{H}^{H}}{I_{M_{r}}\left(p \sigma_{e}^{2}+\sigma_{n}^{2}\right)}\right)\right]\right\} .
\end{aligned}
$$

The lower bound of the ergodic channel capacity is then given as

C

$$
\begin{aligned}
& =E\left\{\log _{2}\left[\operatorname{det}\left(I_{M_{r}}+\frac{p \hat{H} \hat{H}^{H}}{p \sigma_{e}^{2}+\sigma_{n}^{2}}\right)\right]\right\} \\
& =E\left\{\log _{2}\left[\operatorname{det}\left(I_{M_{r}}+\frac{\left(P_{s} / M_{t}\right) \hat{H} \hat{H}^{H}}{\sigma_{n}^{2}} \frac{1}{1+\left(P_{s} / M_{t}\right)\left(\sigma_{e}^{2} / \sigma_{n}^{2}\right)}\right)\right]\right\} \\
& =E\left\{\log _{2}\left[\operatorname{det}\left(I_{M_{r}}+\frac{\rho \hat{H} \hat{H}^{H}}{M_{t}} \frac{1}{1+\left(\rho / M_{t}\right)\left(\mathrm{MSE} / M_{r}\right)}\right)\right]\right\} \\
& =E\left\{\log _{2}\left[\operatorname{det}\left(I_{M_{r}}+\frac{1}{M_{t} / \rho+\mathrm{MSE} / M_{r}} \hat{H} \hat{H}^{H}\right)\right]\right\} .
\end{aligned}
$$

Equation (42) indicates that for a fixed value of $\rho$, the capacity is a function of the estimated channel matrix $\hat{H}$ and the channel estimation MSE.

An uncorrelated Rayleigh channel is ideal. In practical cases, an MIMO channel is usually correlated due to the propagation environment. Consequently, the estimation error matrix is correlated rather than i.i.d. In this case, the variances of elements in the error matrix are not equal to each other and the error correlation matrix is not diagonal. Therefore, (30) and (31) are not true anymore. Thus, (37) needs to be modified as,

$$
\begin{aligned}
u & =\frac{Q \hat{H}^{H}}{\hat{H} Q \hat{H}^{H}+Q R_{e} I_{M_{r}}+\sigma_{n}^{2} I_{M_{r}}} \\
& =\frac{p \hat{H}^{H}}{p \hat{H} \hat{H}^{H}+p R_{e} I_{M_{r}}+\sigma_{n}^{2} I_{M_{r}}} .
\end{aligned}
$$

Then the lower bound capacity (42) becomes,

C

$$
\begin{aligned}
& =E\left\{\log _{2}\left[\operatorname{det}\left(I_{M_{r}}+\frac{p \hat{H} \hat{H}^{H}}{p R_{e}+\sigma_{n}^{2} I_{M_{r}}}\right)\right]\right\} \\
& =E\left\{\log _{2}\left[\operatorname{det}\left(I_{M_{r}}+\frac{\left(P_{s} / M_{t}\right) \hat{H} \hat{H}^{H}}{\sigma_{n}^{2}} \frac{1}{I_{M_{r}}+\left(P_{s} / M_{t} \sigma_{n}^{2}\right) R_{e}}\right)\right]\right\} \\
& =E\left\{\log _{2}\left[\operatorname{det}\left(I_{M_{r}}+\frac{\rho \hat{H} \hat{H}^{H}}{M_{t}} \frac{1}{I_{M_{r}}+\left(\rho / M_{t}\right) R_{e}}\right)\right]\right\} \\
& =E\left\{\log _{2}\left[\operatorname{det}\left(I_{M_{r}}+\frac{1}{\left(M_{t} / \rho\right) I_{M_{r}}+R_{e}} \hat{H} \hat{H}^{H}\right)\right]\right\} .
\end{aligned}
$$

Equation (44) indicates that for a fixed value of $\rho$, the capacity is a function of the estimated channel matrix $\hat{H}$ and the channel estimation error correlation $R_{e}$. As a result, the channel matrix and the quality of channel estimation influence the MIMO capacity.

2.4. MIMO Channel Model. In the undertaken investigations, the Kronecker channel model $[21,22]$ is postulated to construct the channel matrix $H$. In this model, the transmitter and receiver correlation matrices are assumed to be separable and the channel matrix is represented by:

$$
H=R_{R}^{1 / 2} G_{H} R_{T}^{1 / 2},
$$

where $G_{H}$ is the matrix including identical independent distributed (i.i.d) Gaussian entries with the zero mean and the unit variance, and $R_{R}$ and $R_{T}$ are the spatial correlation matrices at the receiver and transmitter, respectively.

Here, it is assumed that the transmitting and receiving sides of MIMO system are equipped with vertically polarized wire dipole antennas. The scattering environment is represented by circles of uniformly distributed scattering objects surrounding the transmitting and receiving nodes. In this case, the spatial correlation matrix elements can be obtained using the Clark's model as given by,

$$
\rho_{i j}^{R(T)}=J_{0}\left(\kappa d_{i j}\right)
$$

where $J_{0}$ stands for the zero-order Bessel function, $\kappa$ is a wave number and $d_{i j}$ is the distance between elements $i$ and $j$ of the uniform array antenna.

The required correlation matrices $R_{T}$ and $R_{R}$ can then be generated using (46)

$$
R_{R(T)}=\left[\begin{array}{ccc}
\rho_{1,1}^{R(T)} & \cdots & \rho_{1, M_{r t}}^{R(T)} \\
\vdots & \ddots & \vdots \\
\rho_{M_{r t}, j}^{R(T)} & \cdots & \rho_{M_{r t}, M_{r t}}^{R(T)}
\end{array}\right]
$$

Having determined $R_{T}$ and $R_{R}$, the channel matrix can be calculated using (45). 


\section{Mutual Coupling Effects}

The mutual coupling in an array of collinear side-by-side wire dipoles can be modeled using the theory described in [27]. Assuming the array is formed by $M_{r t}$ wire dipoles, the mutual matrix can be calculated using the following relationship involving the impedance matrix

$$
\mathbf{C}=\left(Z_{A}+Z_{T}\right)\left(\mathbf{Z}+Z_{T} \mathbf{I}_{M_{r t}}\right)^{-1}
$$

where $Z_{A}$ is the element impedance in isolation, for example, when the wire dipole is $\lambda / 2$, its value is $Z_{A}=73+j 42.5[\Omega]$; $Z_{T}$ is impedance of the receiver at each element chosen as the complex conjugate of $Z_{A}$ to obtain the impedance match. The mutual impedance matrix $\mathbf{Z}$ is given by

$$
\mathbf{Z}=\left[\begin{array}{cccc}
Z_{A}+Z_{T} & Z_{12} & \cdots & Z_{1 M_{r t}} \\
Z_{21} & Z_{A}+Z_{T} & \cdots & Z_{2 M_{r t}} \\
\vdots & \vdots & \ddots & \vdots \\
Z_{M_{r t} 1} & Z_{M_{r t} 2} & \cdots & Z_{A}+Z_{T}
\end{array}\right]
$$

Note that this expression provides the circuit representation for mutual coupling in array antennas. It is valid for single mode antennas. The assumed here wire dipoles fall into this category. For a side-by-side array configuration of wire dipoles having length $l$ equal to $0.5 \lambda$, the expressions for $\left\{\mathbf{Z}_{m n}\right\}$ can be adapted from $[27,28]$ and are rewritten here as,

$$
\begin{aligned}
& \mathbf{Z}_{m n} \\
& = \begin{cases}30\left[0.5772+\ln (2 \kappa l)-C_{i}(2 \kappa l)\right]+j\left[30 S_{i}(2 \kappa l)\right], & m=n, \\
30\left[2 C_{i}\left(u_{0}\right)-C_{i}\left(u_{1}\right)-C_{i}\left(u_{2}\right)\right] & \\
-j\left[30\left(2 S_{i}\left(u_{0}\right)-S_{i}\left(u_{1}\right)-S_{i}\left(u_{2}\right)\right)\right], & m \neq n,\end{cases}
\end{aligned}
$$

where $\kappa$ is the wave number equal to $2 \pi / \lambda$,

$$
\begin{aligned}
& u_{0}=\kappa d_{h}, \\
& u_{1}=\kappa\left(\sqrt{d_{h}^{2}+l^{2}}+l\right) \\
& u_{2}=\kappa\left(\sqrt{d_{h}^{2}+l^{2}}-l\right)
\end{aligned}
$$

$d_{h}$ is the horizontal distance between the two dipole antenna elements. $C_{i}(u)$ and $S_{i}(u)$ are the cosine and sine integrals, respectively. They are given as,

$$
\begin{aligned}
& C_{i}(u)=\int_{\infty}^{u}\left(\frac{\cos (x)}{x}\right) d x \\
& S_{i}(u)=\int_{0}^{\infty}\left(\frac{\sin (x)}{x}\right) d x .
\end{aligned}
$$

In the presence of mutual coupling, the channel matrices $H$ appearing in expression (45) have to be replaced by new channel matrices as given by

$$
H_{\mathrm{mc}}=C_{R} R_{R}^{1 / 2} G_{H} R_{T}^{1 / 2} C_{T}
$$

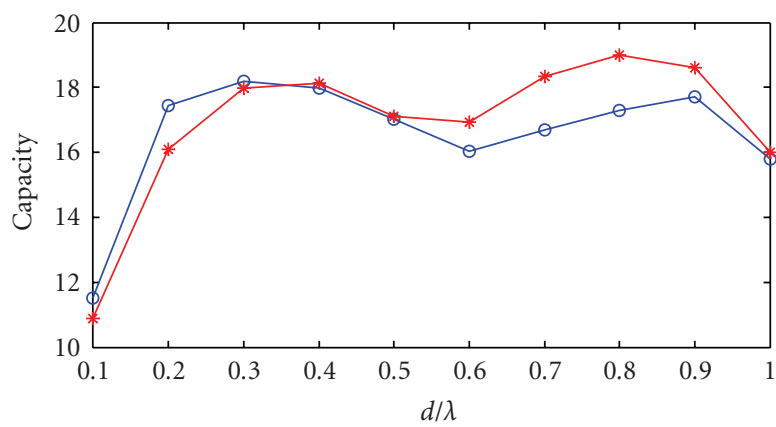

(a) Capacity versus transmitter antenna spacing

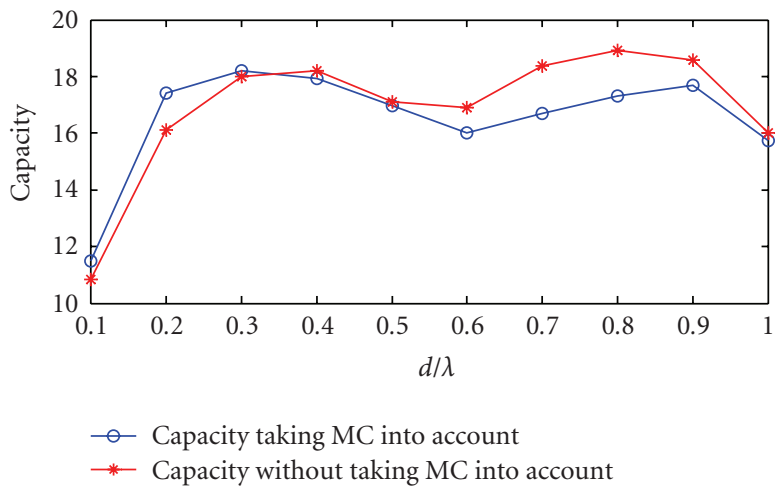

(b) Capacity versus receiver antenna spacing

Figure 1: A $4 \times 4$ MIMO channel capacity considering mutual coupling effect versus transmitter or receiver antenna spacing at SNR equal to $20 \mathrm{~dB}$. (a) Capacity versus transmitter antenna spacing, receiver antenna spacing is set to $1 \lambda$. (b) Capacity versus receiver antenna spacing, transmitter antenna spacing is set to $1 \lambda$.

The modified transmit and receive correlation matrices (including mutual coupling effects) can be expressed by

$$
\begin{aligned}
& R_{R \mathrm{mu}}=C_{R} R_{R}^{1 / 2}, \\
& R_{T \mathrm{mu}}=R_{T}^{1 / 2} C_{T} .
\end{aligned}
$$

Using (54), the expression shown in (45) can be rewritten as (55) and (56), respectively,

$$
\begin{gathered}
H_{\mathrm{mc}}=R_{R \mathrm{mu}} G_{H} R_{T \mathrm{mc}}, \\
R_{H}^{\mathrm{mc}}=H_{\mathrm{mc}}^{H} H_{\mathrm{mc}}=Q_{\mathrm{mc}} \Lambda_{\mathrm{mc}} Q_{\mathrm{mc}}^{H} .
\end{gathered}
$$

Expressions (55) and (56) are the fundamental expressions that can be used to assess the performance of the introduced training-based channel estimation methods and the resulting channel capacity for the MIMO system operating under the condition of mutual coupling being present at transmitter and receiver array antennas.

\section{Simulation Results}

4.1. Perfect CSI Available at Receiver. Figure 1 shows the computer simulation results illustrating the effect of mutual coupling on MIMO channel capacity assuming a perfect 


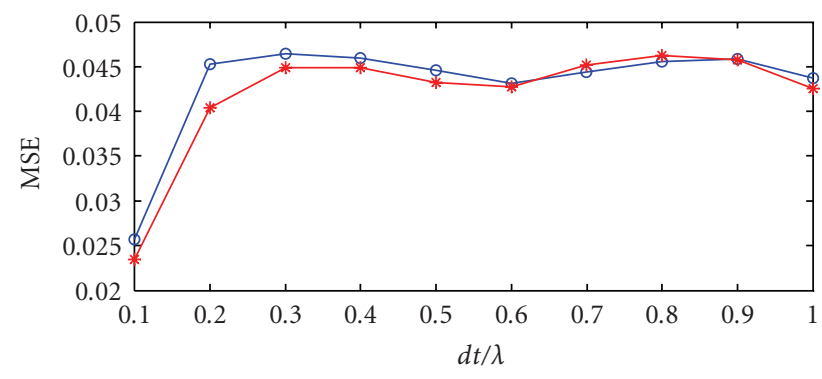

(a) MMSE MSE versus transmitter antenna spacing

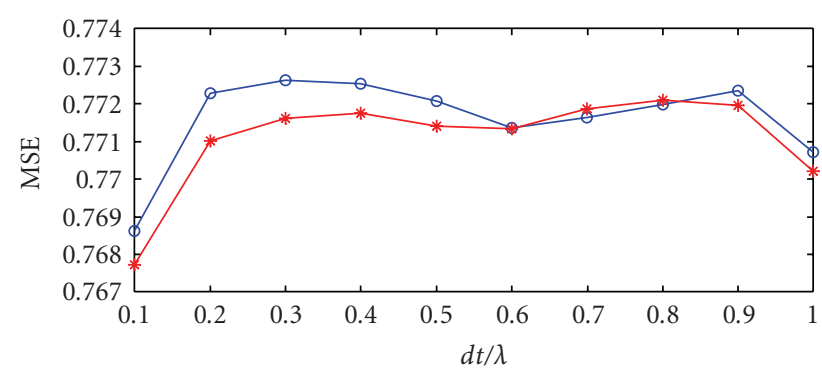

- - MSE taking MC into account
$\rightarrow-$ MSE without taking MC into account

(b) SLS MSE versus receiver antenna spacing

FIGURE 2: MSE of training-based channel estimation for a $4 \times 4$ MIMO considering mutual coupling effect versus transmitter or receiver antenna spacing at SNR equal to $25 \mathrm{~dB}$. (a) MMSE MSE versus transmitter antenna spacing, receiver antenna spacing is set to $1 \lambda$. (b) SLS MSE versus transmitter antenna spacing, receiver antenna spacing is set to $1 \lambda$.

CSI is available at the receiver. In the undertaken computer simulations, both transmitter and receiver are assumed to be equipped with a 4-element uniform array antenna. The elements are assumed to be wire dipoles having length of $0.5 \lambda$. Equations (45) and (55) are used to model the MIMO channel. Both cases when mutual coupling effects are taken into account or disregarded are considered. The transmit SNR is fixed at $20 \mathrm{~dB}$. In the figure, the blue curves represent the case of taking into account mutual coupling effect while the blue curves represent the case when the mutual coupling effect is not taken into account. From the results presented in Figure 1, one can see that when the antenna spacing is smaller than $0.4 \lambda$, the capacity with mutual coupling effect is larger than the one without taking mutual coupling effect into account. When the spacing is larger than $0.4 \lambda$, the channel capacity when mutual coupling effect is taken into account is smaller than when the mutual coupling effect is disregarded. The cross-point occurs at the antenna spacing equal to $0.4 \lambda$. These results show that when the antenna element spacing is within $0.2 \lambda$ and $0.4 \lambda$, the mutual coupling helps to increase the channel capacity when a perfect CSI is assumed to be available at the receiver.

Figure 2 presents the MSE performance of the MMSE and SLS channel estimation methods versus the transmitter antenna element spacing. One can see that MSE for the MMSE method is smaller than for the SLS method. Therefore

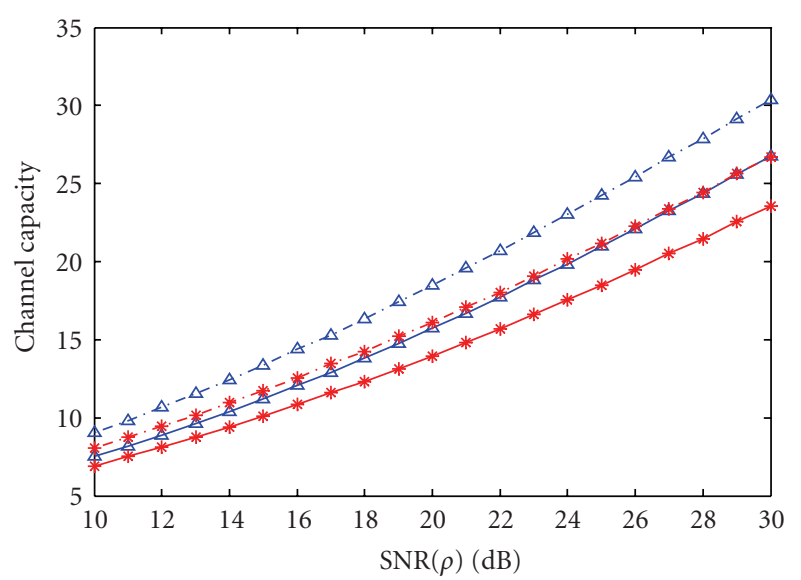

$-\triangle$ - Perfect CSI taking MC into account

$\triangle$ Imperfect CSI taking MC into account

* - Perfect CSI without taking MC into account

* Imperfect CSI without taking MC into account

(a) Channel capacity with esitmation error

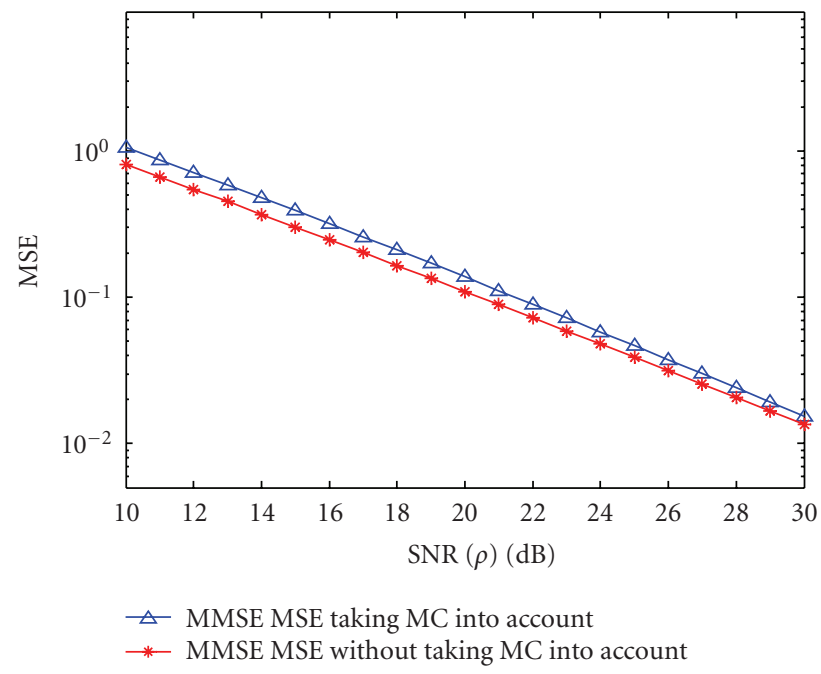

(b) MMSE channel estimation MSE versus SNR

FIGURE 3: (a) Channel capacity considering MMSE estimation error for a $4 \times 4$ MIMO under mutual coupling effect versus SNR. (b) MMSE MSE performance for a $4 \times 4$ MIMO under mutual coupling effect versus SNR; transmitter antenna spacing is set to 0 . $1 \lambda$, receiver antenna spacing is set to $1 \lambda$.

the MMSE shows a superior performance. This agrees with the findings in publications $[16,17]$. On the other hand, for both MMSE and SLS methods, an apparent difference in MSE occurs when mutual coupling effects are taken into account or disregarded when the transmitter antenna element spacing varies from $0.1 \lambda$ to $0.5 \lambda$. It is apparent that MSE when mutual coupling effects are taken into account is worse than the one when the mutual coupling is disregarded (not taken effect into account). When the antenna element spacing is larger than $0.5 \lambda$, the difference disappears. This finding agrees with the prediction that when the antenna element spacing is between $0.2 \lambda$ to $0.4 \lambda$ the mutual coupling compromises the accuracy of MIMO channel estimation. 
4.2. Imperfect CSI due to Channel Estimation Errors. This time, computer simulations for MIMO channel capacity include both mutual coupling effects as well as channel estimation errors. MMSE method is assumed to be used to estimate the channel matrix (CSI). Again, both the transmitter and receiver are assumed to be equipped with 4-element uniform array antennas. The antenna elements are assumed to be wire dipoles having length of $0.5 \lambda$. The antenna spacing at the receiver is set to $1 \lambda$ while the one at transmitter is assumed to be $0.2 \lambda$.

Figure 3(a) shows the results for the $4 \times 4$ MIMO channel capacity assuming perfect and imperfect CSI at the receiver. One can see that when the channel estimation error is taken into account, the channel capacity is lower than the one assuming a perfect CSI at the receiver. Also, at the transmitter antenna element spacing equal to $0.2 \lambda$, the channel capacity taking into account the mutual coupling effect is always higher than when the mutual coupling is neglected. Figure 3(b) shows the results for MSE of MMSE method for the $4 \times 4$ MIMO system when mutual coupling effect is included or disregarded. One can see that for both cases, the MSE decreases as SNR becomes larger. One can see that MSE becomes worse when mutual coupling effect is included. The results presented in Figures 3(a) and 3(b) show that although the mutual coupling undermines the channel estimation accuracy, it does not adversely affect the channel capacity. For the presented cases, the mutual coupling decreasing the spatial correlation level improves the signal transmission quality. Therefore, the worsening channel estimation has only an insignificant impact on capacity.

\section{Conclusion}

In this paper, the effect of mutual coupling on MIMO channel estimation accuracy and the resulting channel capacity has been investigated. The mathematical analysis and simulation results have shown that when the antenna element spacing at either transmitter or receiver is within $0.2 \lambda$ and $0.4 \lambda$, the mutual coupling decreases the spatial correlation level and undermines the estimation accuracy of the MIMO channel. However, the reduced accuracy of channel estimation does not have a significant impact on the channel capacity. The carried out computer simulations have shown that the decreased spatial correlation improves the capacity of MIMO system despite having an adverse effect on the channel estimation.

\section{References}

[1] E. Telatar, "Capacity of multi-antenna Gaussian channels," European Transactions on Telecommunications, vol. 10, no. 6, pp. 585-595, 1999.

[2] C. Budianu and L. Tong, "Channel estimation for spacetime orthogonal block codes," IEEE Transactions on Signal Processing, vol. 50, no. 10, pp. 2515-2528, 2002.

[3] T. L. Marzetta and B. M. Hochwald, "Capacity of a mobile multiple-antenna communication link in rayleigh flat fading,"
IEEE Transactions on Information Theory, vol. 45, no. 1, pp. 139-157, 1999.

[4] A. Narula, M. J. Lopez, M. D. Trott, and G. W. Wornell, "Efficient use of side information in multiple-antenna data transmission over fading channels," IEEE Journal on Selected Areas in Communications, vol. 16, no. 8, pp. 1423-1436, 1998.

[5] A. Grant, "Joint decoding and channel estimation for linear MIMO channels," in Proveedings of the IEEE Wireless Communications and Networking Conference, pp. 1009-1012, Chicago, III, USA, September 2000.

[6] A. S. Kyung, R. W. Heath, and H. K. Baik, "Shannon capacity and symbol error rate of space-time block codes in MIMO rayleigh channels with channel estimation error," IEEE Transactions on Wireless Communications, vol. 7, no. 1, pp. 324-333, 2008.

[7] X. Zhang and B. Ottersten, "Performance analysis of V-BLAST structure with channel estimation errors," in Proceedings of the IEEE 4th Workshop on Signal Processing Advances in Wireless Communications (SPAWC '03), pp. 487-491, June 2003.

[8] P. Layec, P. Piantanida, R. Visoz, and A. O. Berthet, "Capacity bounds for MIMO multiple access channel with imperfect channel state information," in Proceedings of the IEEE Information Theory Workshop (ITW'08), pp. 21-25, May 2008.

[9] T. Svantesson and A. Ranheim, "Mutual coupling effects on the capacity of multi-element antenna systems," in Proceedings of the Acoustics, Speech, and Signal Processing (ICASSP'01), vol. 4, pp. 2485-2488, April 2001.

[10] J. Wallace and M. Jensen, "The capacity of MIMO wireless systems with mutual coupling," in Proceedings of the IEEE Vehicular Technology Conference (VCT '02), pp. 696-700, Vancouver, Canada, 2002.

[11] J. Wallace and M. Jensen, "Mutual coupling in MIMO wireless systems: a rigorous network theory analysis," IEEE Transactions on Wireless Communications, vol. 3, no. 4, pp. 1317-1325, 2004.

[12] P. N. Fletcher, M. Dean, and A. R. Nix, "Mutual coupling in multielement array antennas and its influence on MIMO channel capacity," IEEE Electronics Letters, vol. 39, no. 4, pp. 342-344, 2003.

[13] S. Lu, H. T. Hui, M. E. Bialkowski, and X. Liu, "Optimizing signal transmission in a MIMO system influenced by antenna mutual coupling," The ECTI Transactions on Electrical Engineering, Electronics, and Communications, vol. 6, no. 2, pp. 7378, 2008.

[14] S. Lu, X. Liu, H. T. Hui, M. Bialkowski, N. Seman, and H. Y. Zhang, "Power allocation strategy for compact MIMO transmitters with uniform circular arrays under the influence of antenna mutual coupling," in Priceedings of the Asia-Pacific Microwave Conference Proceedings (APMC '07), December 2007.

[15] S. Lu, H. T. Hui, M. Bialkowski, and X. Liu, "Optimizing transmission strategy in single-user MIMO systems under the influence of antenna mutual coupling," in Proceedings of the International Symposium on Communications and Information Technologies (ISCIT '07), pp. 370-374, October 2007.

[16] M. Biguesh and A. B. Gershman, "MIMO channel estimation: Optimal training and tradeoffs between estimation techniques," in Proceedings of the IEEE International Conference on Communications (ICC '04), vol. 5, pp. 2658-2662, Paris, France, June 2004.

[17] M. Biguesh and A. B. Gershman, "Training-based MIMO channel estimation: a study of estimator tradeoffs and optimal 
training signals," IEEE Transactions on Signal Processing, vol. 54, no. 3, pp. 884-893, 2006.

[18] X. Liu, M. E. Bialkowski, F. Wang, et al., "Investigation into the effect of spatial correlation on channel estimation and capacity of multiple input multiple output system," International Journal of Communications, Network and System Sciences, vol. 2, no. 4, pp. 267-275, 2009.

[19] X. Liu, M. E. Bialkowski, and F. Wang, "Investigation into effects of channel properties on trainning-based MIMO channel estimation," ECTI Transaction on Electrical Engineering, Electronics and Communications, vol. 20, no. 2, 2010.

[20] X. Liu, M. E. Bialkowski, and S. Lu, "Investigations into training-based MIMO channel estimation for spatial correlated channels," in Proceedings of the IEEE Antennas and Propagation Society (APS '07), pp. 205-208, Kohala Coast, Hawaii, USA, 2007.

[21] E. G. Larsson and P. Stoica, Space-Time Block Coding for Wireless Communication, Cambridge University Press, Cambridge, UK, 2003.

[22] C. N. Chuah, D. N. C. Tse, J. M. Kahn, and R. A. Valenzuela, "Capacity scaling in MIMO wireless systems under correlated fading," IEEE Transactions on Information Theory, vol. 48, no. 3, pp. 637-650, 2002.

[23] S. M. Kay, Fundamentals of Statistic Signal Processing: Estimation Theory, Prentice-Hall, Englewood Cliffs, NJ, USA, 1993.

[24] M. Torabi, M. R. Soleymani, and S. Aissa, "On the performance of MIMO-OFDM systems with imperfect channel information," in Proceedings of the International Conference on Wireless Networks, Communications and Mobile Computing, vol. 1, pp. 600-605, June 2005.

[25] M. Medard, "The effect upon channel capacity in wireless communications of perfect and imperfect knowledge of the channel," IEEE Transactions on Information Theory, vol. 46, no. 3, pp. 933-946, 2000.

[26] T. Yoo and A. Goldsmith, "Capacity of fading MIMO channels with channel estimation error," in Proceedings of the IEEE International Conference on Communications, vol. 2, pp. 808813, 2004.

[27] S. Durrani and M. E. Bialkowski, "Effect of mutual coupling on the interference rejection capabilities of linear and circular arrays in CDMA systems," IEEE Transactions on Antennas and Propagation, vol. 52, no. 4, pp. 1130-1134, 2004.

[28] M. E. Bialkowski, P. Uthansakul, K. Bialkowski, and S. Durrani, "Investigating the performance of MIMO systems from an electromagenetic perspective," Microwave and Optimal Technology Letters, vol. 48, no. 7, pp. 1233-1238, 2006. 

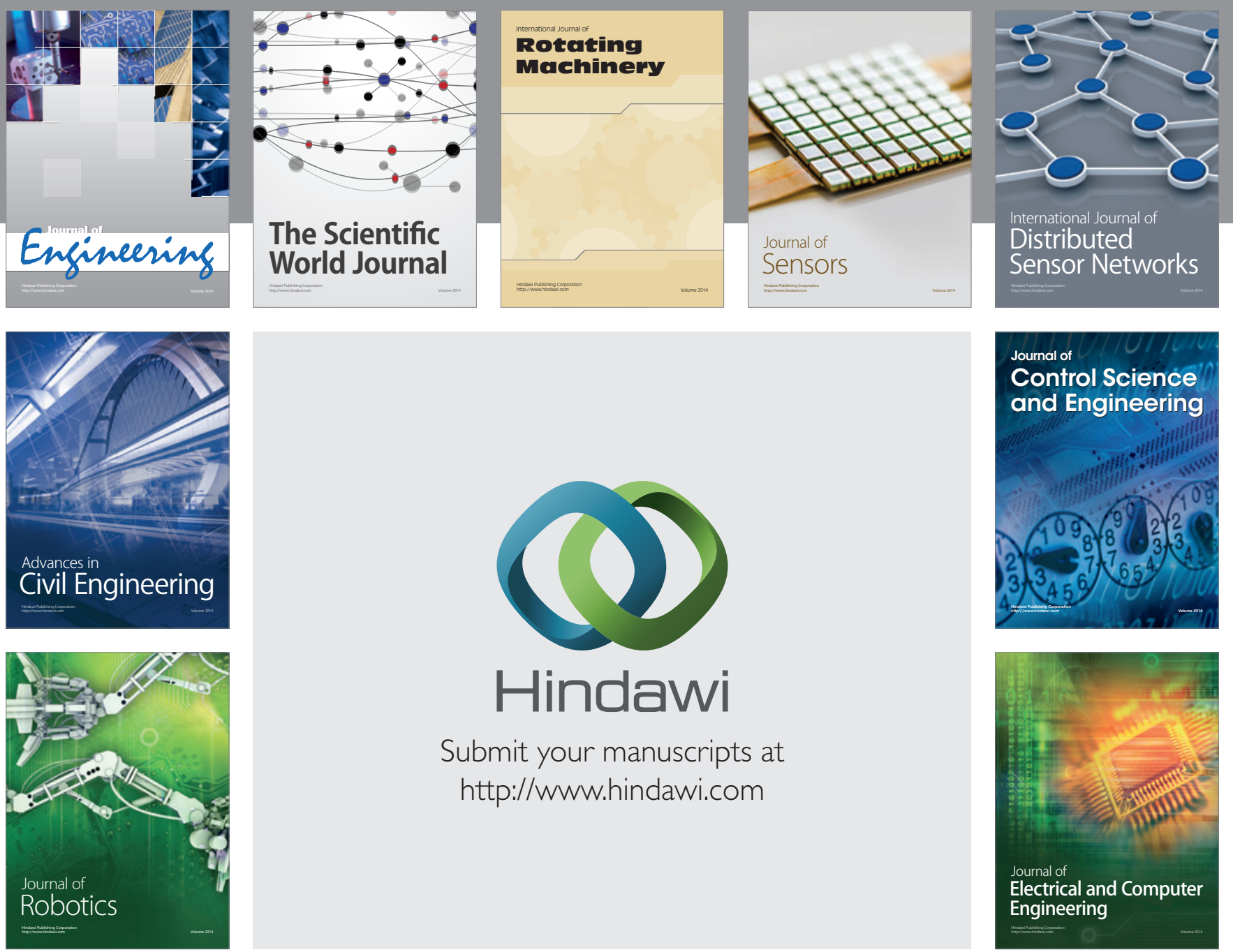

Submit your manuscripts at

http://www.hindawi.com
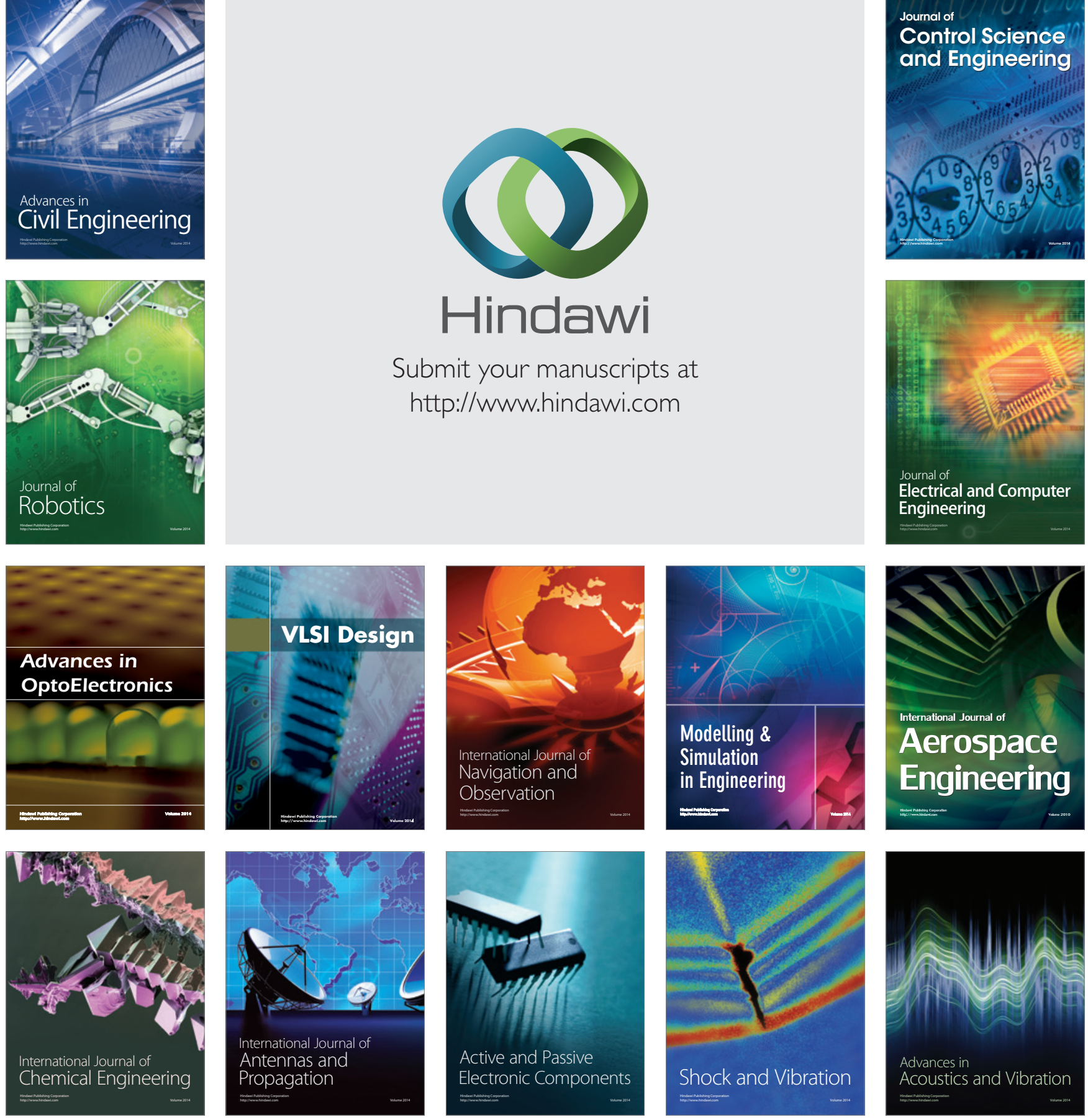